\title{
The Distinct Element Method - Application to Structures in Jointed Rock
}

\author{
J. Morris, L. Glenn, S. Blair, F. Heuze
}

This article was submitted to International Workshop Meshfree Methods of Partial Differential Equations, Bonn, Germany, September 11-14, 2001

\section{November 30, 2001}

U.S. Department of Energy

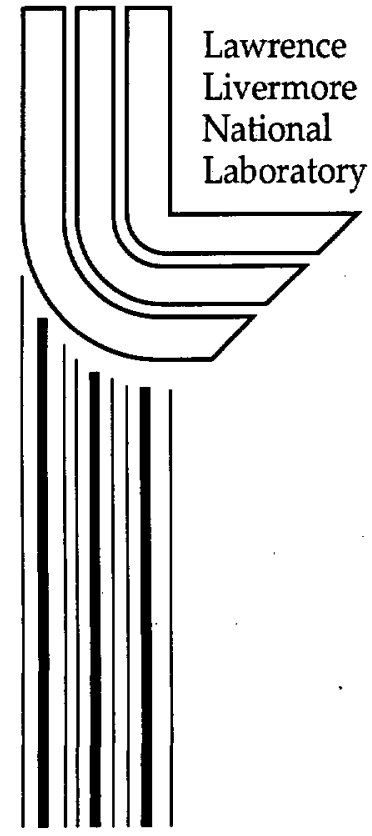




\section{DISCLAIMER}

This document was prepared as an account of work sponsored by an agency of the United States Government. Neither the United States Government nor the University of California nor any of their employees, makes any warranty, express or implied, or assumes any legal liability or responsibility for the accuracy, completeness, or usefulness of any information, apparatus, product, or process disclosed, or represents that its use would not infringe privately owned rights. Reference herein to any specific commercial product, process, or service by trade name, trademark, manufacturer, or otherwise, does not necessarily constitute or imply its endorsement, recommendation, or favoring by the United States Government or the University of California. The views and opinions of authors expressed herein do not necessarily state or reflect those of the United States Government or the University of California, and shall not be used for advertising or product endorsement purposes.

This is a preprint of a paper intended for publication in a journal or proceedings. Since changes may be made before publication, this preprint is made available with the understanding that it will not be cited or reproduced without the permission of the author.

This report has been reproduced directly from the best available copy.

Available electronically at http://www.doe.gov/bridge

Available for a processing fee to U.S. Department of Energy and its contractors in paper from

U.S. Department of Energy

Office of Scientific and Technical Information

P.O. Box 62

Oak Ridge, TN 37831-0062

Telephone: (865) 576-8401

Facsimile: (865) 576-5728

E-mail: reports@adonis.osti.gov

Available for the sale to the public from

U.S. Department of Commerce

National Technical Information Service 5285 Port Royal Road

Springfield, VA 22161

Telephone: (800) 553-6847

Facsimile: (703) 605-6900

E-mail: orders@ntis.fedworldigov

Online ordering: http://www.ntis.gov/ordering.htm

OR

-Lawrence Livermore National Laboratory

Technical Information Department's Digital Library

http://www.llnl.gov/tid/Library.html 


\title{
The Distinct Element Method - Application to Structures in Jointed Rock
}

\author{
Joseph Morris, Lew Glenn, Steve Blair, and Francois Heuzé \\ Geophysics and Global Security Division, Lawrence Livermore National \\ Laboratory, Livermore, U.S.A.
}

\begin{abstract}
The Distinct Element Method (DEM) is a meshfree method with applications to rock mechanics, mining sciences, simulations of nuclear repositories, and the stability of underground structures.

Continuum mesh-based methods have been applied successfully to many problems in geophysics. Even if the geology includes fractures and faults, when sufficiently large length scales are considered a continuum approximation may be sufficient. However, a large class of problems exist where individual rock joints must be taken into account. This includes problems where the structures of interest have sizes comparable with the block size. In addition, it is possible that while the structure may experience loads which do no measurable damage to individual blocks, some joints may fail. This may launch smaller blocks as dangerous projectiles or even cause total failure of a tunnel. Traditional grid-based continuum approaches are wholly unsuited to this class of problem. It is possible to introduce discontinuities or slide lines into existing grid-based methods, however, such limited approaches can break down when new contacts form between blocks.

The distinct element method (DEM) is an alternative, meshfree approach. The DEM can directly approximate the block structure of the jointed rock using arbitrary polyhedra. Using this approach, preexisting joints are readily incorporated into the DEM model. In addition, the method detects all new contacts between blocks resulting from relative block motion.

We will describe the background of the DEM and review previous application of the DEM to geophysical problems. Finally we present preliminary results from a investigation into the stability of underground structures subjected to dynamic loading.
\end{abstract}

\section{Numerical Simulation of Discontinua}

A wide range of applications involve materials or systems which are discontinuous at some level. While some systems may be intrinsically discontinuous (such as items of furniture in a room) other discontinuous systems are well approximated by a continuum. Soils, for example, are clearly discontinuous at the microscale, however, in practice, the behavior of soils are well represented by continuum methods. This approximation is possible because the scale of the objects of interest (dams, walls, etc.) is large compared with a typical soil grain. There are also applications where a discontinuous system may be approximated better by a continuum or discontiuum depending upon 
the specific information sought. For example, continuum mesh-based methods have been applied successfully to many problems in geophysics. Even if the geology includes fractures and faults, when sufficiently large length scales are considered a continuum approximation may be sufficient. However, a large class of problems exist where individual rock joints must be taken into account. This includes problems where the structures of interest have sizes comparable with the block size. In addition, it is possible that while the structure may experience loads which do no measurable damage to individual blocks, some joints may fail. A continuum, mesh-bashed treatment of such systems is usually inappropriate.

\section{Discrete Element Methods}

Cundall and Hart, 1992 review many of numerical techniques that have been developed to simulate the behavior of discontinuous systems. Cundall and Hart, 1992 define a discrete element method to have the following properties:

- Allows finite displacements and rotations of discrete bodies, including complete detachment

- Recognizes new contacts automatically as the calculation progresses.

Cundall and Hart, 1992 further define four basic classes of discrete element methods:

- Distinct element methods (DEM)

- Modal methods

- Discontinuous deformation analysis

- Momentum-exchange methods

Distinct element methods (DEM) use an explicit scheme to evolve the equations of motion of discrete bodies directly. The bodies may be rigid or deformable (by subdivision into elements). Early approaches employed rigid disks or spheres with compliant contacts (Cundall and Strack, 1979, Cleary, 1991). Cundall, 1980, Cundall and Hart, 1985 and Walton, 1980 developed 2-dimensional DEMs which employed arbitrary polygons. More recently, fully three-dimensional DEMs with rigid or deformable polyhedral blocks with compliant contacts were developed (Cundall, 1988, Hart el al., 1988), When using the DEM, contacts are always deformable and can employ detailed joint constitutive models which mimic observed fracture properties (normal stiffness, shear stiffness, dilation, cohesion, etc.). Quasi-static analysis can be performed by relaxation of the body motion. The DEM is considered in more detail in the following section.

Modal methods (Williams et al., 1985, Williams and Mustoe, 1987) are similar to the distinct element method in the case of rigid blocks. However, for deformable bodies, modal superposition is used. This method appears to be better suited for loosely-packed discontinua. In dynamic simulation of dense 
packings, eigenmodes are not revised to account for additional contact constraints.

In contrast with the Distinct element method, Discontinuous deformation analysis (Shi, 1988 and Shi and Goodman, 1988) assumes contacts are rigid. The bodies may be rigid or deformable and the condition of no-penetration is achieved by an iterative scheme. Deformability is achieved through superposition of strain modes.

Momentum-Exchange methods (Hahn, 1988) assume both the contacts and bodies to be rigid. Collisions are modeled by an instantaneous exchange of momentum between contacting bodies. It is possible to include friction sliding in the contact model.

\section{Distinct Element Method}

The Distinct element method (DEM) can directly approximate the block structure of the jointed rock using arbitrary polyhedra. Using this approach, preexisting joints are readily incorporated into the DEM model. By nature, the Distinct element method can readily handle large deformation on the joints. In addition, the method detects all new contacts between blocks resulting from relative block motion. The Lagrangian nature of the DEM simplifies tracking of material properties as blocks of material move. It is also possible to guarantee exact conservation of linear and angular momentum. Furthermore, by using an explicit integration scheme, the joint models can be very flexible. In particular, the joint constitutive model can incorporate experimentally observed effects such as, cohesion, joint dilation, and friction angle.

The DEM has been applied to a wide range of problems in geomechanics. For example, Antonellini and Pollard, 1995 simulated the formation of shear bands in sandstone using the DEM. Morgan, 1999a,b applied the DEM to the mechanics of granular shear zones. Heuzé et al., 1993 used the DEM to analyze explosions in hard rock. Sanderson and Zhang, 1998 used a twodimensional distinct element method to investigate the evolution of damage zones and fluid flow in fracture networks and around faults. Cundall, 2001 reviews the application of the DEM to simulation of granular material and rock.

The DEM has also been coupled to other techniques in order to exploit the relative advantages (accuracy vs. speed) of each in different regions of the computational domain. For example, Lorig et al., 1986 used a hybrid model that incorporated both distinct element and boundary element methods (BEM) to analyze the behavior of jointed rock. The DEM was used to simulate the near-field behavior in detail, while the BEM provided boundary conditions that simulated the far-field rock mass. Pan and Reed, 1991 presented details of a coupled distinct element-finite element method that was successfully applied to several field-scale test problems. Mori, Otsu, and Osakada, 1997 
used a viscoplastic finite-element method to generate boundary conditions for a DEM simulation of grain alignment in the "mushy-state" forging of magnets. In addition, the DEM has been applied to simulate the transient dynamics of fracturing solids. Munjiza, Owen, and Bicanic, 1995 developed a coupled finite discrete-element technique where deformable discrete elements were allowed to separate into two or more elements once a critical stress (or strain) state was reached. Sawamoto et al., 1998 used a simple DEM with cylindrical elements to assess local damage to reinforced concrete.

The flexible nature of the DEM presents a few challenges. The algorithm for detecting an classifying contacts must be both robust and efficient. Realistic three-dimensional fracture networks require a great many DEM blocks and concomitant computational expense. These two issues are discussed in the following sections.

\subsection{Detecting Contacts}

As the distinct element blocks move, initial contacts between blocks may disappear, and new contacts form. The DEM code must rapidly determine if two blocks are interacting. In addition, the code must be able to classify the type of contact so an appropriate constitutive model may be applied. For example, the behavior of a vertex pushing into a face may differ qualitatively from two faces in contact. The method used should be able to identify all conceivable cases of two block interaction. In particular, near degenerate cases, such as two faces barely overlapping, should not cause the algorithm to fail.

One approach is to exhaustively consider the arrangement of two blocks by looking at each pair of vertices. If the blocks have $m$ and $n$ vertices each, this direct approach requires order $m n$ operations to determine if two blocks interact. In addition, the algorithm in three dimensions is very complex and requires many different cases to be identified and treated appropriately. Ghaboussi et al., 1993 proposed training neural networks to perform contact detection. While neural networks are relatively simple to implement they require extensive training.

The concept of a "Common-Plane" (Cundall, 1988) provides an alternative approach to reduce the complexity of the contact detection algorithm. Intuitively, the approach can be thought of as introducing a rigid, massless plate between candidate interacting pairs of convex polyhedra (see Figure 1). The algorithm proceeds by iterating the position and orientation of this "common-plane" to maximize the distance between it and each block. If the blocks are overlapping, the common-plane will orient to minimize the penetration of the blocks through the common-plane. Figure 2 shows some examples of how the common-plane is oriented for two-dimensional polygonal elements. Of particular interest is the treatment of non-convex blocks. In this case the block must be subdivided into convex polyhedra and common-planes are generated for each pair of convex polyhedra belonging to separate blocks. 
The common-plane can be used to simplify contact classification. Cundall, 1988 suggest classifying the contact type by simply counting up the number of interacting vertices on each block. For example, two penetrating vertices from one block and three from the other would indicate an edge interacting with a face. In addition, the common-plane simplifies the application of joint constitutive models. For example, the orientation of the common-plane can be used to determine the normal of the contact and dictates a co-ordinate system which can be used by joint models.
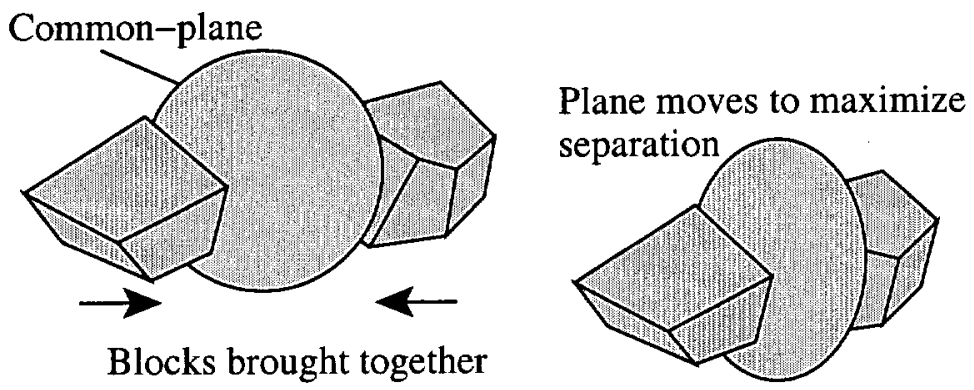

Fig. 1. The "common-plane" can be thought of as a rigid, massless plate between candidate interacting pairs of convex polyhedra. The algorithm proceeds by iterating the position and orientation of this "common-plane" to maximize the distance between it and each block.

The iterative procedure of the common-plane approach is very easy to implement and can be very efficient for many classes of problem. Typically, the common-plane orientation from the previous time-step provides a good initial guess of the current orientation. Provided the appropriate normal of the contact has not changed much between time steps, the iterative procedure converges rapidly.

\subsection{Parallelization of DEM}

The number of distinct elements used in a single simulation is limited by the available computational power. Current work stations can handle simulations involving of order one hundred thousand elements. Several authors have extended the capability of the DEM by utilizing high performance computing (HPC) systems. Cleary and Sawley, 1999 and Sawley and Cleary, 1999 have developed a portable parallel implementation of the DEM using standard message passing libraries. Horner et al., 1998 and Carrillo et al., 1999 recently implemented a fully interactive large-scale soil simulation involving hundreds of thousands of elements. Dowding et al., 1999 developed a parallel DEM to calculate the dynamic response of a cavern in a jointed rock mass. 

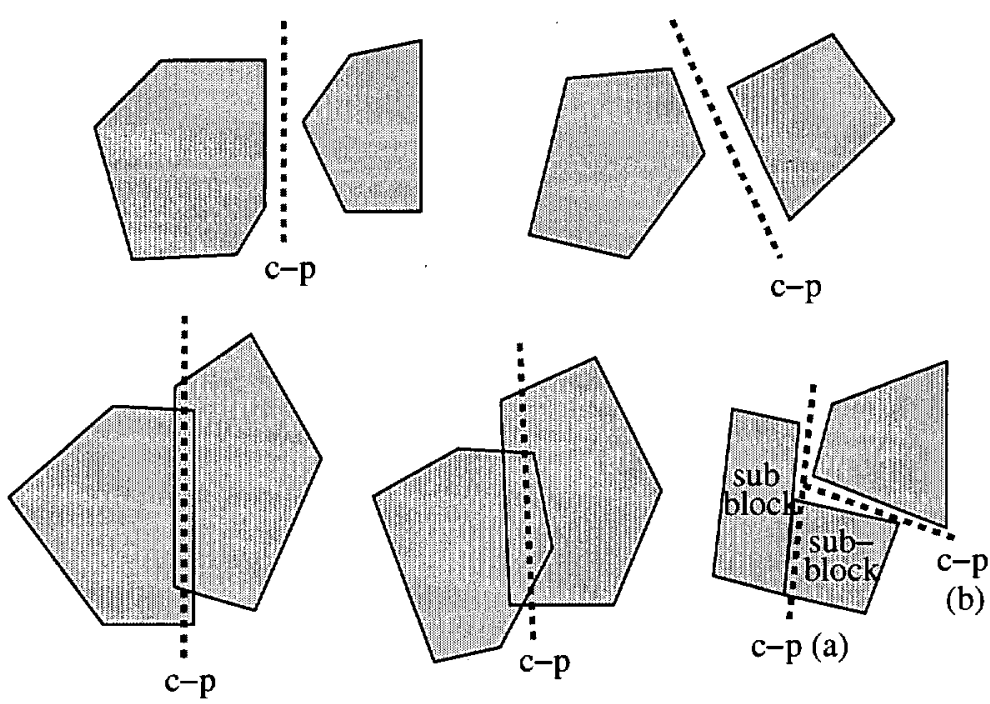

Fig. 2. Some examples of how the common-plane is oriented for two-dimensional polygonal elements. The last example shows the treatment of non-convex blocks. The block is subdivided into convex polyhedra and a common-plane is generated for each pair of convex polyhedra belonging to separate blocks.

They compared SIMD (single instruction, multiple data) performance with MIMD (multiple instruction, multiple data) and found MIMD processing to provide the best overall parallelization. Ghaboussi et al., 1993 proposed an alternative parallelization scheme on SIMD architectures by using parallelized neural networks to perform the contact detection. However, to date this approach does not appear to have been implemented.

Our approach was to parallelize through spatial domain decomposition. The entire problem domain is divided into nearest neighbor cells which are used to identify neighboring blocks which are potential contacts. Each processor is assigned a contiguous region of nearest neighbor cells. Communication occurs via message passing (MPI) at the start of each time step. All blocks within neighboring cells are copied between processors. Calculations are performed on blocks which do not directly interact with neighboring processors while communication occurs. Figure 3 shows the performance of our parallel code for increasing problem sizes and increasing numbers of processors. Duplicate calculations are performed on each processor in the region of overlap where blocks are copied back and forth. Consequently, speedup is best for larger problems where the region of overlap between processors is a smaller fraction of the total work performed. 


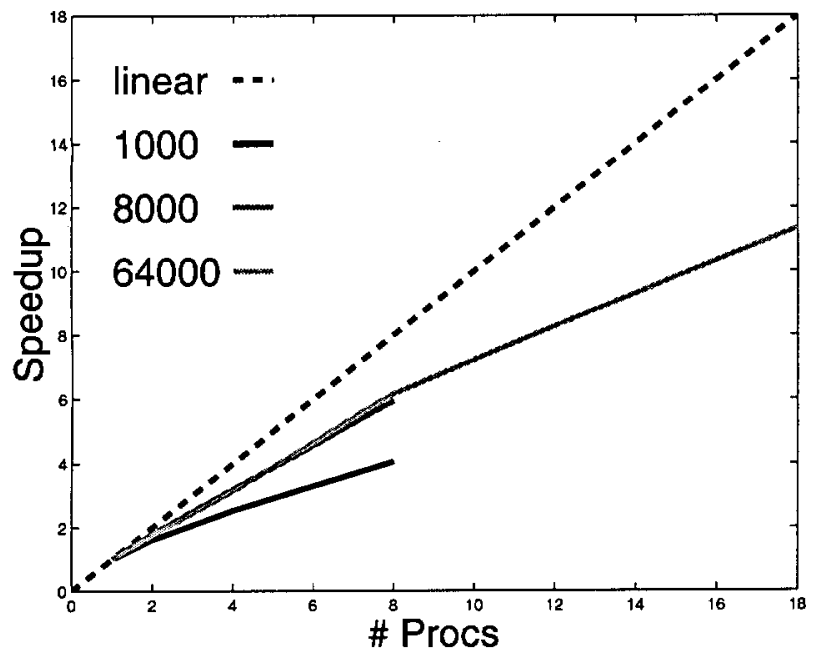

Fig. 3. Performance of our parallel code for increasing problem sizes and increasing numbers of processors. Speedup is best for larger problems where the region of overlap between processors is a smaller fraction of the total work performed.

\section{Attack/Defense of Buried Facilities}

The particular application of interest is the prediction of damage to hard and deeply buried targets (HDBT), such as command and control centers, or chemical, biological, and nuclear weapons facilities. Figure 4 depicts three scenarios for attacking an HDBT:

1. Direct blast into the entrance passageway.

2. Collapsing a critical access point with a single explosion.

3. Several blasts to a point well above the facility.

To predict damage inflicted upon hard and deeply buried targets, several coupled regions must be modeled (see Figure 5). In the immediate vicinity of the blast, the ground shock is sufficient to rubblize the rock, material strength is irrelevant, and the material behavior is hydrodynamic. Deeper into rock, material strength becomes important. Finally, in the vicinity of the target area, the detailed structure of the rock mass and the facility itself are important.

\subsection{Continuum Approach}

An estimate of peak velocity, displacement, and stress experienced at a given depth from an explosion can be used as an estimator of damage done to deeply buried tunnels. Typically, the depth of the tunnel is large compared 


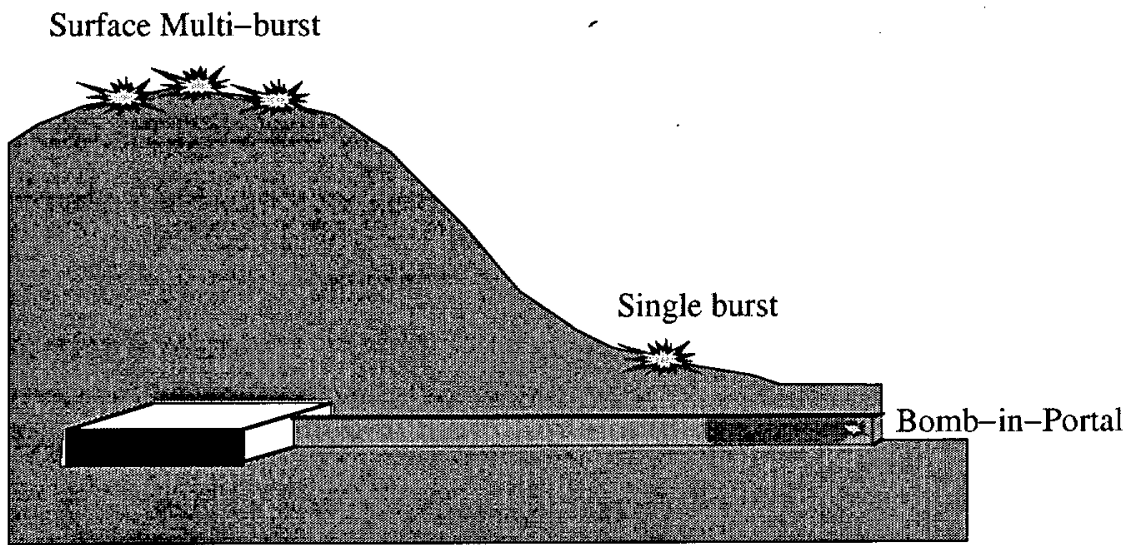

Fig. 4. Three possible scenarios for attacking a hard and deeply buried target: (1) direct blast into the entrance passageway, (2) collapsing a critical access point with a single explosion, or (3) several blasts to a point well above the facility.

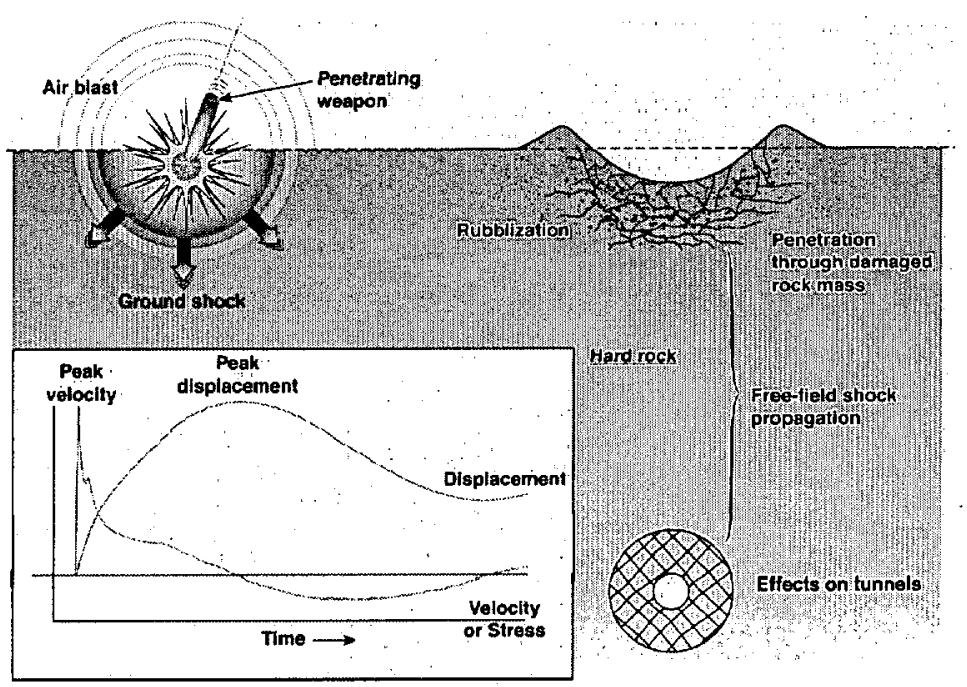

Fig. 5. To predict damage inflicted upon hard and deeply buried targets, several coupled regions must be modeled. In the immediate vicinity of the blast, the ground shock is sufficient to rubblize the rock, material strength is irrelevant, and the material behavior is hydrodynamic. Deeper into rock, material strength becomes important. Finally, in the vicinity of the target area, the detailed structure of the rock mass and the facility itself are important. 
with the size of the blocks making up the rock, and continuum approaches have been very successful in reproducing measured attenuation rates from the source. Lomov et al., 2001 present an approach for accurately modeling projectile penetration and explosions in rock media. Using an Eulerian code (GEODYN) Lomov et al., 2001 fit a constitutive model (Rubin et al., 2000) to peak velocity and displacement attenuation data from tamped (buried) nuclear explosions in hard rock conducted in the U. S. and in the Hoggar massif (Algeria). The model was then validated with tamped data from nuclear explosions at Degelen Mountain. The predicted attenuation rates for peak velocity and displacement were also in good agreement with those measured in 500-ton surface HE shots at Degelen Mountain. This continuum



Fig. 6. Constitutive Model fit to ensemble of nuclear tamped explosion (peak velocity/displacement) data

treatment demonstrates that peak velocity and displacement from tamped explosions in granitic rocks can be predicted to within a factor of two over ten orders of magnitude in yield (see Fig. 6). However, this approach does not provide insight into the details of the damage done to underground structures. Traditionally a rock mass is deemed to fail when the strength of the material is exceeded. Failed rock is no longer able to withstand load without undergoing inelastic strains. However, hard rock strength increases markedly with increased pressure and yet it has been observed that functional damage or even complete tunnel collapse can occur at stress levels far below those 
previously thought to be required. The following section investigates this phenomenon.

\subsection{Results from Underground Explosions}

Recent experiments have suggested that repeated loading of hard and deeply buried targets by, for example, multiple bombing runs may cause functional damage or even complete tunnel collapse at stress levels far below those previously thought to be required. Similar behavior was also observed at the Nevada Test Site during nuclear tests. In this section we discuss some observations of tunnels in Granite and Tuff from the Nevada Test Site, subjected to various peak stresses. The Pile Driver and Midas Myth tests were performed in granite and tuff respectively. The tunnel sections varied widely in their degree of rock mass reinforcement and tunnel lining or support. In the cases presented here, it was reported that no damage had taken place in the granite material where the free field stress had not exceeded $700 \mathrm{MPa}$. Some sections withstood free-field stresses in excess of $200 \mathrm{MPa}$, whereas some others were damaged at less than $100 \mathrm{MPa}$. Well engineered tunnel designs can withstand much higher loads than unsupported or unreinforced sections.

Figure 7 compares two different sized tunnel sections within granite, reinforced with concrete. Under approximately the same loading conditions, the larger construction failed. Figure 8 compares two tunnel segments in granite with rock bolt and wiremesh reinforcement. Both were subjected to approximately $100 \mathrm{MPa}$ peak stress and the tunnel segment orientated side-on to the source failed. Figure 9 shows the collapse of two excavations in tuff which were subjected to loads well below the strength of tuff. Both structures failed, and the discrete nature of the rock mass is evident.

In all these cases, the peak stress experienced by the structure was well below the strength of the intact rock, and yet many of these structures failed. The mechanism for this damage is thought to be key-block displacement. To comprehend how this works it must first be understood that the structure surrounding an underground tunnel does not normally consist of homogeneous rock. Instead the rock is typically jointed via naturally occurring faults or cracks. The crack density may be enhanced by the very process that forms the tunnel, e.g., drill and blast. The resulting blocks are held in place by frictional forces and/or cementation contacts. When the ensemble block structure is subject to intense impulsive loading it is possible that one or a few (key) blocks on the tunnel wall can be displaced enough to release the equilibrium state on a large section of blocks. The key block(s) then can fall into the open tunnel and the space previously occupied by these blocks becomes available for occupation by adjacent blocks; this process can lead to catastrophic collapse of the entire roof of the tunnel. Even if this does not occur, the structure can be seriously weakened and subsequent loading may cause collapse at much lower loading. 
These results also indicate that engineered structures play a key role. Furthermore, the orientation, spacing, and shear strength of geologic discontinuities (joints) control the behavior of a tunnel of a given diameter. Hard rock joints dilate strongly before reaching peak strength, after which the strength drops rapidly with increased loading. As a result of the controlling effects of the joints it is not possible to estimation tunnel response via continuum based analysis. While it is possible to augment continuum approaches to handle fractures and faults by introducing discontinuities or slide lines into existing grid-based methods, this approach becomes increasingly difficult if the connectivity of the block structures changes.

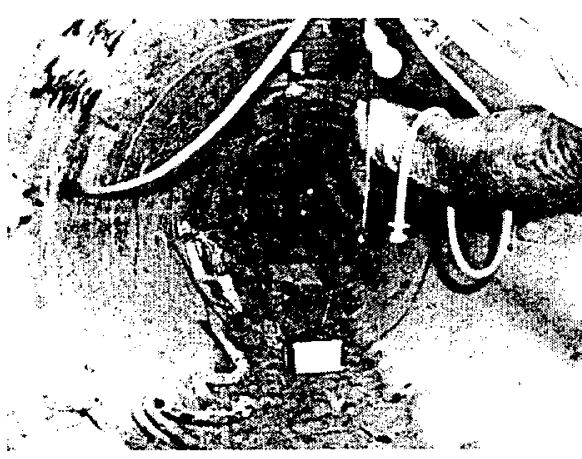

(a)

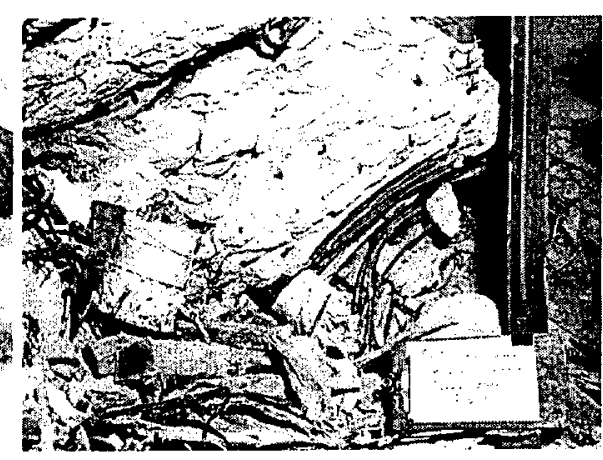

(b)

Fig. 7. (a) A $7 \mathrm{ft}$ inner diameter tunnel in concrete inside a backpacked $13 \mathrm{ft}$ diameter excavation within granite. Subjected to over $200 \mathrm{MPa}$, the tunnel suffered minimal damage. (b) A similar, but larger construction (12'x7' backpacked, reinforced concrete intersecting with a $26.7^{\prime} \times 16^{\prime}$ 'rock excavation) failed under comparable load.

\section{Application of DEM to Structures in Hard Rock}

\subsection{Wave Propagation}

In this section we demonstrate the ability of the DEM to solve wave propagation problems before using the DEM to simulate the response of realistic tunnel geometries in jointed rock. Figure 10 shows idealized pulse, similar to that experienced at some depth below a surface explosion. This pulse was driven into a regular lattice of cubic DEM blocks of side length $1 \mathrm{~m}$, with normal stiffness of $3 \times 10^{10} \mathrm{~Pa} / \mathrm{m}$ and density of $2 \times 10^{3} \mathrm{~kg} / \mathrm{m}^{3}$. The pulse is also shown after it has been transmitted through $2001 \mathrm{~m}$ DEM blocks. In this case, the joint model was elastic, so no energy is lost and the pulse peak 


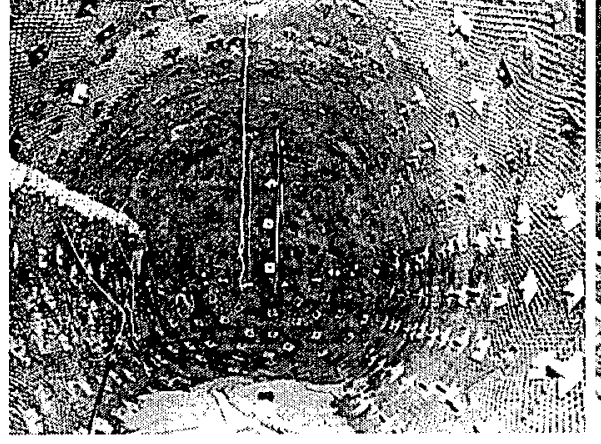

(a)

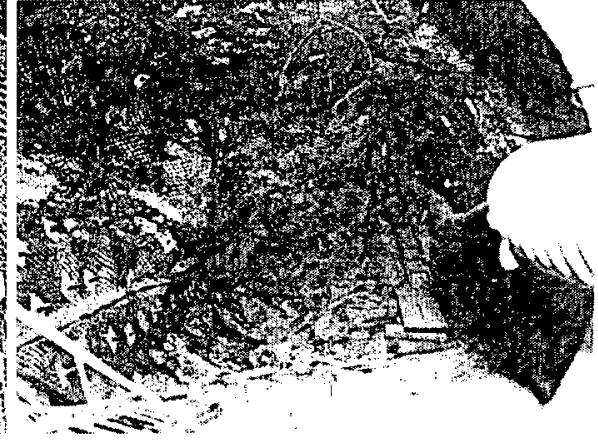

(b)

Fig. 8. (a) A 16-ft diameter tunnel with no liner, with rock bolts and wiremesh reinforcing within granite. Subjected to over $100 \mathrm{MPa}$ end-on loading, the tunnel suffered essentially no damage. (b) The same basic design, oriented with side-on loading failed under the same load. The failed block structure can be discerned through the wire mesh.

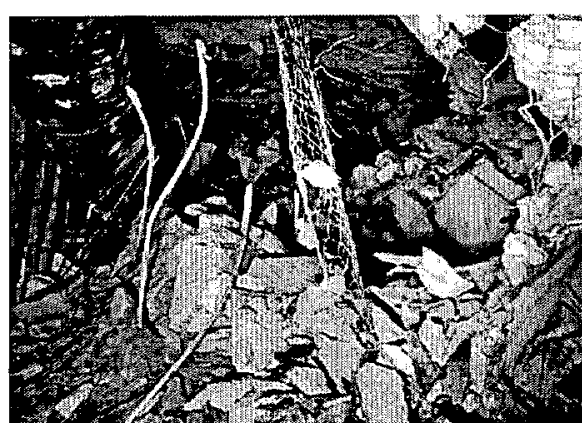

(a)

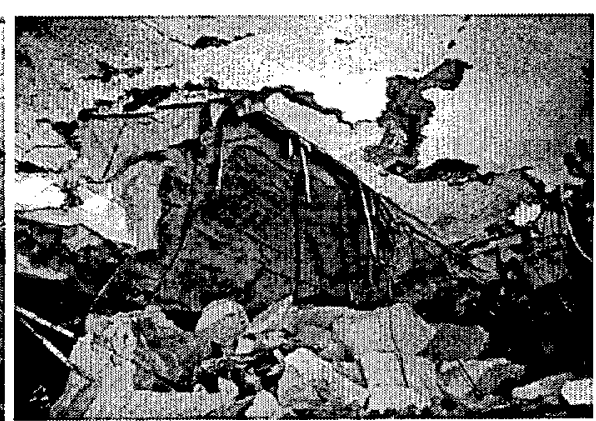

(b)

Fig. 9. (a) An excavation, reinforced with wire within tuff, collapsed at low stress. (b) An excavation, reinforced with rockbolts within tuff, collapsed at low stress. 
is propagated with negligible attenuation. The expected wave speed is given by:

$$
c=\sqrt{l K_{n} / \rho}
$$

where $l$ is the length of each block in the propagation direction, $\rho$ is the block density, and $K_{n}$ is the normal stiffness of the contacts. The simulation exhibits a wave speed of $3820 \mathrm{~m} / \mathrm{s}$ which compares well with the analytic result of $3873 \mathrm{~m} / \mathrm{s}$. Note that while this test assumed elastic joint models, hysteretic contact forces have been considered by other authors (Heuzé et al., 1993) and will be incorporated into our code in the near future.

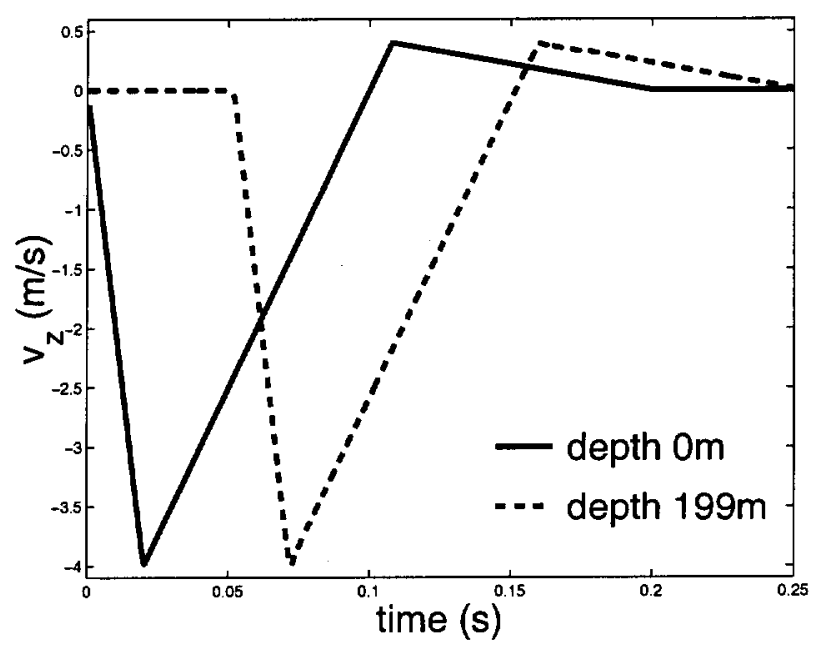

Fig. 10. A idealized typical velocity profile is well propagated through a stack of $2001 \mathrm{~m}$ DEM blocks.

\subsection{Simulations of Tunnels}

Finally we present preliminary results from a investigation into the stability of underground structures subjected to dynamic loading. The effects of explosions on underground structures were studied in two dimensions by Heuzé et al., 1993. Figure 11 shows a tunnel in jointed rock. The average block size is approximately $1 \mathrm{~m}$. The jointed rock mass is confined by $7.5 \mathrm{MPa}$ of lateral and vertical stress. The upper boundary was moved with the driving pulse shown in Fig. 10.

The simulation predicts that a substantial portion of the tunnel roof will collapse, making the tunnel unusable. Although this simulation is preliminary, 
it demonstrates the capabilities of the DEM with this kind of problem. The joint structure is realistic, with non-orthogonal joint planes. Blocks are free to move and make new contacts with other blocks in the simulation. After further validation, this simulation capability will be applied to a parameter study of the stability of underground structures.
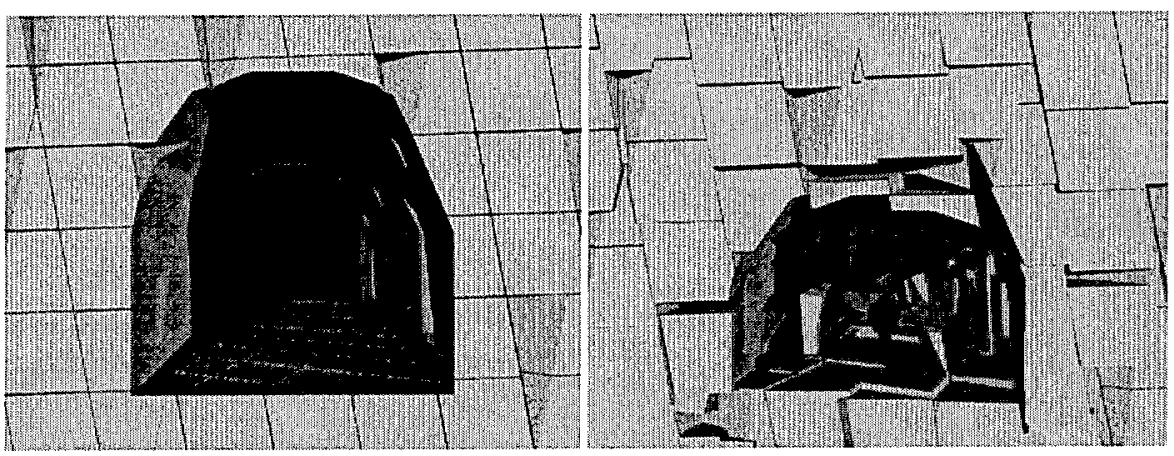

Fig. 11. A preliminary simulation of a tunnel in jointed rock subjected to the pulse shown in Fig. 10. The simulation predicts that a substantial portion of the tunnel roof will collapse, making the tunnel unusable.

\section{Discussion}

We have seen that continuum approaches can provide some details of potential damage to underground structures. Peak velocities and displacements are well predicted to within a factor of two over ten orders of magnitude in yield. However, this information along cannot provide complete damage estimates. In particular, it has been observed that substantial damage, or total collapse of a tunnel can occur at stresses well below the strength of the rock. The mechanism for this damage is thought to be key-block displacement. Realistic simulation of failure Distinct element methods can simulate collapse of tunnels for realistic fracture set geometry including the effects of block motion.

In practice, however, we only have limited knowledge of local fault zones. To provide bounds on the response, we must study a range of probable fault geometries. That is, we must perform stochastic analysis with many realizations required to obtain adequate statistics to bound results. Current three-dimensional scalar codes take days to simulate the dynamic loading of structures containing $\sim 10^{4}-10^{5}$ distinct elements. We have presented our approach to parallelization of the DEM by employing domain decomposition.

The common-plane approach to contact detection is both efficient and easy to implement. However, under some circumstances the method of Cundall, 1988 
gives misleading contact classifications. The contact type is based upon counting the number of penetrating vertices. Thus, it is possible that a contact which would be better approximated by two interpenetrating faces will be misclassified as an edge to face contact. We will be investigating more accurate contact detection algorithms which build on the common-plane approach. Other future development of our DEM code will include coupling with other codes and the inclusion of structural elements. In addition, more advanced joint models including hysteresis (Heuzé et al., 1993) will be incorporated into our code in the near future.

Acknowledgement This work was performed under the auspices of the U.S. Department of Energy by the University of California, Lawrence Livermore National Laboratory under Contract No. W-7405-Eng-48.

\section{References}

Antonellini M.A., Pollard D.D. (1995) Distinct element modeling of deformation bands in sandstone. J. Struct. Geol., 17:1165-1182

Carrillo A.R., West J.E., Horner D.A., Peters J.F. (1999) Interactive large-scale soil modeling using distributed high performance computing environments. Int. J. High Perf. Comput. Appl., 13:1:33-48

Cleary P.W. (1991) Extensions of the hybrid method for granular flows. In: Proc. 5th International Computational Techniques and Applications Conference, Adelaide, Australia

Cleary P.W., M.L. Sawley (1999) Three-dimensional modelling of industrial granular flows. In: Second International Conference on CFD in the Minerals and Process Industries, CSIRO, Melbourne, Australia, 95-100

Cundall P.A. (1980) UDEC-A generalized distinct element program for modelling jointed rock, Final Tech. Rep. Eur. Res. Office (US Army Contract DAJA3779-C-0548); NTIS order No. AD-A087 610/2

Cundall P.A. (1988) Formulation of a three-dimensional distinct element modelPart I. A scheme to detect and represent contacts in a system composed of many polyhedral blocks. Int. J. Rock Mech. Min. Sci. \& Geomech. Abstr. 25:107-116

Cundall P.A. (2001) A Discontinuous Future for Numerical Modelling in Geomechanics? Geotech. Eng., 149:1:41-47

Cundall P.A., Hart R.D. (1985) Development of generalized 2-D and 3-D disinct element programs for modeling jointed rock, Misc. Paper SL-85-1, US Army Corps of Engineers

Cundall P.A., Hart D.H. (1992) Numerical Modelling of Discontinua. Eng. Comput., 9:101-113

Cundall P.A., Strack O.D.L. (1979) A discrete numerical model for granular assemblies. Géotechnique, 29:47-65

Dowding C.H., Dmytryshyn O., Belytschko T.B. (1999) Parallel processing for a discrete element program Comput. Geotech. 25:4:281-285

Ghaboussi J., Basole M.M., Ranjithan S. (1993) Three-dimensional discrete element analysis on massively parallel computers. In: Second International Conference on Discrete Element Methods, MIT, Cambridge, MA 
Hahn J.K. (1988) Realistic animation of rigid bodies. Comp. Graph. 22:299-308

Hart R., Cundall P.A., Lemos J. (1988) Formulation of a Three-dimensional Distinct Element Model- Part II. Mechanical Calculations for Motion and Interaction of a System Composed of Many Polyhedral Blocks. Int. J. Rock Mech. Min. Sci. \& Geomech. Abstr. 25:117-125

Heuzé F.E., Walton O.R., Maddix D.M., Shaffer R.J., Butkovich T.R. (1993) Analysis of Explosions in Hard Rocks: The Power of Discrete Element Modeling. In: Hudson J.A., Brown E.T., Fairhurst C., Hoek E. (Eds.) Comprehensive Rock Engineering, Vol. 2, Analysis and Design Methods, Pergamon Press, 387-413

Horner D.A., Carrillo A.R., Peters J.F., West J.E. (1998) High resolution soil vehicle interaction modeling. Mech. Struct. \& Mach., 26:3:305-318

Lomov I., Antoun T., Glenn L. (2001) Explosion in the granite field: Hardening and softening behavior in rocks. In: Proceedings of 12th APS Topical Conference, Shock Compression of Condensed Matter, June 24-29, Atlanta, Georgia

Lorig L.J., Brady B.H.G., Cundall P.A. (1986) Hybrid distinct element-boundary element analysis of jointed rock. Int. J. Rock. Mech. Min. Sci. \& Geomech. Abstr., 23:4:303-312

Morgan J.K. (1999) Numerical simulations of granular shear zones using the distinct element method 1. Shear zone kinematics and the micromechanics of localization. J. Geophys. Res., 104:B2:2703-2719

Morgan J.K. (1999) Numerical simulations of granular shear zones using the distinct element method 2. Effects of particle size distribution and interparticle friction on mechanical behavior. J. Geophys. Res., 104:B2:2721-2732

Mori K., Otsu M., Osakada K. (1997) Distinct element simulation of grain alignment in mushy-state forging of magnets. Int. J. Mech. Sci., 39:7:771-780

Munjiza A., Owen D.R.J., Bicanic N. (1995) A combined finite-discrete element method in transient dynamics of fracturing solids. Eng. Comput. 12:145-174

Pan X.D., Reed, M.B. (1991) A coupled distinct element-finite element method for large deformation analysis of rock masses. Int. J. Rock Mech. Min. Sci. \& Geomech. Abstr. 28:1:93-99

Rubin M.B., Vorobiev O.Y., Glenn L.A. (2000) Mechanical and numerical modeling of a porous elastic-viscoplastic material with tensile failure. International Journal of Solids and Structures 37:1841-1871

Sanderson D.J., Zhang X. (1998) Deformation, damage and fluid flow in fracture networks and around faults. Fall Meeting of the American Geophysical Union.

Sawamoto Y., Tsubota H., Kasai Y., Koshika N., Morikawa H. (1998) Analytical studies on local damage to reinforced concrete structures under impact loading by discrete element method. Nucl. Eng. Des., 179:157-177

Sawley M.L., Cleary P.W. (1999) A parallel discrete element method for industrial granular flow simulations. EPFL Supercomputing Review 11:23-29

Shi Gen-Hua, (1988) Discontinuous deformation analysis- a new numerical model for the statics and dynamics of block systems. PhD Thesis, University of California, Berkeley

Shi Gen-Hua, Goodman R.E. (1988) Discontinuous deformation analysis- a new method for computing stress, strain and sliding of block systems. In: Cundall P.A. et al. (Eds.) Key Questions in Rock Mechanics, Balkema, Rotterdam, 381-383

Walton O.R. (1980) Particle dynamics modeling of geological materials, Rep. UCRL-52915, Lawrence Livermore National Laboratory 
Williams J.R., Hocking G., Mustoe G.G.W. (1985) The theoretical basis of the discrete element method. In: NUMETA ' 85 , Balkema, Rotterdam

Williams J.R., Mustoe G.G.W. (1987) Modal methods for the analysis of discrete systems, Comp. Geotechnics 4:1-19 